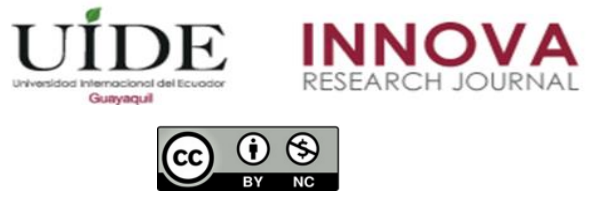

INNOVA Research Journal, ISSN 2477-9024

(Mayo-Agosto 2021). Vol. 6, No.2 pp. 255-273

DOI: https://doi.org/10.33890/innova.v6.n2.2021.1553

URL: http://revistas.uide.edu.ec/index.php/innova/index

Correo: innova@uide.edu.ec

\title{
Percepción de la comunidad con respecto al Plan Regional de Acción Ambiental (2017-2021). Caso Región Ucayali- Callería- Perú
}

\section{Community perception regarding the Regional Environmental Action Plan (2017-2021). Ucayali-Callería- Perú Region Case}

Ederlinda Ortega Casique

Universidad César Vallejo, Perú

ederlindaortegac@hotmail.com

(D) https://orcid.org/0000-0001-7396-3316

Recepción: 11/11/2020 | Aceptación: 03/01/2021 | Publicación: 10/05/2021

Cómo citar (APA, séptima edición):

Ortega Casique, E. (2021). Percepción de la comunidad con respecto al Plan Regional de Acción Ambiental (2017-2021). Caso Región Ucayali-Callería-Perú. Innova Research Journal, 6(2), 255-273. https://doi.org/10.33890/innova.v6.n2.2021.1553

\section{Resumen}

La globalización en que vivimos ha generado que los países del mundo estén preocupados en temas ambientales y sus consecuencias. El Perú no es una excepción es por ello, el objetivo del presente artículo pretende la mejora del Plan Regional de Acción Ambiental (2017- 2021). Caso UcayaliCallería- Perú; propone acciones para fortalecerla. Estudio de enfoque cualitativo, básico, método hermenéutico, diseño estudio de caso, mediante la entrevista y el Focus Group, utilizando la guía semiestructurada, validado por expertos investigadores; se transcribió, codificó, analizó, categorizó y ordenó. Según la revisión y análisis basado en las Líneas estratégicas, se encontró, que del total de acciones solo una está dedicada a las comunidades nativas siendo insuficiente y no inclusiva; con planificación lenta, acciones temporales y presupuesto insuficiente. Las subcategorías emergentes del estudio, Herencia territorial, Asociatividad y Bien común, permitió proponer acciones desde propiciar el cambio de mentalidad en las comunidades nativas; implementar canales de comunicación necesarios en promover sus productos y servicios como resultado de la asociatividad con el empresariado en los proyectos productivos con involucramiento de los actores, sin presionar los sistemas ecológicos naturales existentes, para lograr el desarrollo con dignidad y solidaridad en las comunidades nativas.

Palabras claves: líneas estratégicas; comunidades nativas; herencia territorial; asociatividad; bien común. 
Ederlinda Ortega Casique.

ISSN 2477-9024. Innova Research Journal (Mayo-Agosto, 2021). Vol 6, No. 2, pp. 255-273

\begin{abstract}
The globalization in which we live has caused the countries of the world to be concerned about environmental issues and their consequences, Peru is not an exception; therefore, the objective of this article aims to improve the Regional Environmental Action Plan (2017-2021). UcayaliCalleria- Peru Case; proposes actions to strengthen it. Qualitative, basic approach study, hermeneutical method, case study design, through the interview and the Focus Group, using the semi-structured guide, validated by expert researchers; it was transcribed, coded, analyzed, categorized and ordered. According to the review and analysis based on the Strategic Lines, it was found that of the total of actions, only one is dedicated to native communities, being insufficient and not inclusive; with slow planning, temporary actions and insufficient budget. Focused on the Research Line Strengthening the Actors of the Economy and Business Development in the section of politics and government that is the vocation and concern of the magazine, as well as spreading knowledge in people and organizations. The emerging subcategories of the study, Territorial inheritance, Associativity and Common Good, allowed to propose actions from promoting a change of mentality in native communities; Implement the necessary communication channels to promote their products and services as a result of associativity with the business community in productive projects with the involvement of the actors, without putting pressure on the existing natural ecological systems to achieve development with dignity and solidarity in the native communities.
\end{abstract}

Keywords: strategic lines; native communities; territorial heritage; associativity; common good.

\title{
Introducción
}

El presente estudio referido al tema medioambiental es un tema global que interesa a todos los que vivimos en el planeta tierra y cada país adopta las medidas en cuanto a políticas y normativas. Las organizaciones a nivel mundial como el BID, CEPAL, PNUMA, etc., son los responsables de solucionar los problemas ambientales y asegurar el futuro sostenible. En Europa se ha venido trabajando en este tema desde varias décadas atrás, desde elaborar normativas para el cuidado del medio ambiente articulando políticas educativas en todas las instituciones de educación y comunidades. En América también se ha venido adoptando medidas para combatir la contaminación e investigando los efectos positivos y negativos producidos por el hombre y la sociedad y planteando soluciones ante el mundo científico y político. Lo que significa "el cambio y toma de conciencia" de las personas para acceder al bienestar presente y futuro.

En el Perú se planteó solucionar el problema medioambiental con la elaboración de diversos planes como el "Plan Bicentenario hacia el 2021" basado en la Declaración Universal de los Derechos Humanos; el Plan Estratégico de Desarrollo Nacional (PEDN) con enfoque de Desarrollo Humano de las Naciones Unidas, teniendo como referentes los Objetivos del milenio, la Constitución de la República del Perú 1979, las políticas del Acuerdo Nacional con objetivos, indicadores, metas, programas y estrategias para que éstas sean cumplidas en todos los niveles de gobierno.

A nivel regional se contó con el Plan Regional de Desarrollo Concertado de la región Ucayali (PRDC), con enfoque climático, orientado a la gestión e integrado por todos los actores 
regionales buscando la sostenibilidad y optimización de los recursos económicos de la región, sirvió de base para el Plan Regional de Acción Ambiental (PRAA), es el instrumento de planificación regional de mediano plazo de la jurisdicción de la región Ucayali, cuenta con siete líneas estratégicas que fundamentan la investigación en esta zona productora de agua, alimento y otros recursos, con una población de más de medio millón de personas que precisan de grandes cantidades de agua, suelo y aire.

La región Ucayali, es la segunda región amazónica más extensa del Perú y representa el 7.97\% del territorio peruano, situado en la zona central y oriental del territorio peruano, tiene una superficie de 102,410.18 km2 y aproximadamente 600,000 habitantes. La región tiene cuatro provincias y 15 distritos: la provincia competencia del estudio es la provincia de Coronel Portillo que tiene 6 distritos: Callería, Manantay, Campoverde, Yarinacocha, Nuevo Requena y Masisea. Según la Intergovermental Science Police Plattform on Biodiversity and Ecosytem Services (IPBES, 2019) en la Amazonía se vienen incrementando las actividades de extracción y explotación de los RRNN, ocasionando la extinción de las especies, otras causas son los incendios forestales, pérdidas de hectáreas de bosque, pesca excesiva, caza, que destruyen los hábitats naturales. Ante esta situación problemática es necesario tomar acciones, planificarlos y cumplirlos porque satisface las necesidades de la población usando la naturaleza y haciéndola sostenible, para asegurar la vida y economía del lugar.

Se justifica teóricamente en los conceptos teóricos que fundamentan el estudio cualitativo basado en las definiciones apriorísticas de las líneas estratégicas del PRAA y tiene varias perspectivas como resultado. Presenta justificación metodológica sustentada en el diseño de caso que proviene de su propia realidad. Además, contiene justificación práctica porque origina una propuesta de acciones como solución del vacío encontrado como producto del análisis y revisión realizado.

Ante ello, la formulación del problema general fue el siguiente: ¿Cuál es la percepción de la comunidad con respecto al Plan Regional de Acción Ambiental (2017-2021).caso Región Ucayali- Callería-Perú? y como problemas específicos: a) ¿Cuál es la percepción de la comunidad con respecto al PRAA en la Línea Estratégica 1: Educación y Gobernanza (2017-2021) en UcayaliCallería?, b)¿Cuál es la percepción de la comunidad con respecto al PRAA en la Línea Estratégica 2: Reforestación (2017-2021) en Ucayali-Callería?, c) ¿Cuál es la percepción de la comunidad con respecto al PRAA en la Línea Estratégica 3: Minería y Energía (2017-2021) en Ucayali- Callería?, d) ¿Cuál es la percepción de la comunidad con respecto al PRAA en la Línea Estratégica 4: Manejo de Residuos sólidos (2017-2021) en Ucayali-Callería?, e) ¿Cuál es la percepción de la comunidad con respecto al PRAA en la Línea Estratégica 5: Gestión de la Calidad del Agua (2017-2021) en Ucayali- Callería?, f)¿Cuál es la percepción de la comunidad con respecto al PRAA en la Línea Estratégica 6: Diversidad Biológica (2017-2021) en Ucayali- Callería?, g) ¿Cuál es la percepción de la comunidad con respecto al PRAA en la Línea Estratégica 7: Aire (2017-2021) en UcayaliCallería?

Los objetivos generales y específicos fueron: Determinar la percepción de la comunidad con respecto al PRAA (2017-2021). Caso Región Ucayali- Callería-Perú. a)Determinar la percepción de la comunidad con respecto al PRAA en la Línea Estratégica 1: Educación y 
Ederlinda Ortega Casique.

ISSN 2477-9024. Innova Research Journal (Mayo-Agosto, 2021). Vol 6, No. 2, pp. 255-273

Gobernanza (2017-2021) en Ucayali-Callería, b) Determinar la percepción de la comunidad con respecto al PRAA en la Línea Estratégica 2: Reforestación (2017-2021) en Ucayali-Callería, c) Determinar la percepción de la comunidad con respecto al PRAA en la Línea Estratégica 3: Minería y Energía (2017-2021) en Ucayali- Callería, d) Determinar la percepción de la comunidad con respecto al PRAA en la Línea Estratégica 4: Manejo de Residuos sólidos (2017-2021) en Ucayali-Callería, e) Determinar la percepción de la comunidad con respecto al PRAA en la Línea Estratégica 5: Gestión de la Calidad del Agua (2017-2021) en Ucayali- Callería, f) Determinar la percepción de la comunidad con respecto al PRAA en la Línea Estratégica 6: Diversidad Biológica (2017-2021) en Ucayali- Callería, g) Determinar la percepción de la comunidad con respecto al PRAA en la Línea Estratégica 7: Aire (2017-2021) en Ucayali- Callería.

\section{Marco teórico}

Los estudios de la Red internacional Published on Servindi (2015); Organismo de Evaluación y Fiscalización Ambiental (OEFA, 2018) e IPBES (2019) publicaron la preocupación de los países y de la sociedad mundial, con respecto a los ecosistemas existentes en el mundo, deteriorados por el clima o por el ser humano y propusieron serios debates, mesas redondas en diferentes países para obtener toda la información que suceda en el país y que es común a los intereses públicos y privados para proponer mejoras, recomendar, priorizar su importancia y aplicación en su dimensión ambiental.

Otros estudios internacionales como la revista catalana De Dret Ambiental destaca las investigaciones de Boto y Mellado (2018) refirieron que existen pocos avances en normativas en el principado de Asturias en los residuos y contaminación atmosférica y dieron mayor importancia a otros temas y propuestas de protección y control ambiental industrial, actuación de funcionarios medioambientales y plantearon estrategias en el uso de venenos y el uso de recursos hídricos y una reforma interna en las normativas del suelo y uso.

Además, los trabajos especializados desarrollados por Tabares y Vera (2017); Martínez y Ortiz (2019) y los realizados en Nicaragua, Colombia y Brasil según Amador y Mendoza (2018) y Zúñiga (2018); Monteiro , Partidario y Meuleman (2018); Santos ( 2017); Cortés (2019) coincidieron en manifestar su compromiso de responsabilidad y cuidado del MA, cumplimiento de compromisos internacionales del derecho sostenible adoptados en RIO+20 y COP21 y el desarrollo de políticas, planes y programas de protección , manejo, conservación de las áreas del Estado y señala que el desarrollo sostenible debe ser evaluado por cada país, para la toma de decisiones respetando los valores culturales e institucionales que influyen en la interpretación y resultados. Santos (2017) refirió que la gobernanza influye operativamente buscando la calidad de bienes y servicios ambientales, si hacemos el uso correcto, repercutirá en el nivel cultural de las comunidades del contexto y promoverá el futuro del mundo y garantizará el existir de la humanidad presente y futura. Cortés (2019) resaltó su preocupación por el medio ambiente incluyendo el agua, efectos y erosiones que arriesgan el paisaje e indicó qué hacer para prevenirlos.

A nivel nacional hay poca información con respecto al tema, entre las investigaciones encontradas se tienen las investigaciones realizadas por Chiarelli (2018); Caicedo y Morvelli (2016); los realizados por García, Inga y Párraga (2018); los aportes de Echevarría, Guillinta, 
Sifuentes y Zapata (2018) propusieron el método del Planeamiento Estratégico para sus regiones según normativas peruanas definieron la importancia en seguir el proceso estratégico. Chiarelli (2018) se propuso obtener la crítica al método y expuso el proceso del planeamiento competitivo entre los distritos, provincias y regiones, etc. basados en el diagnóstico y objetivos de desarrollo humano.

Según la Constitución Política del Perú (2013) y el Ministerio del Ambiente (MINAM, 2013) Ley 28611, señala que toda acción Estado-Sociedad centra a la persona humana como fin superior y prioriza este derecho para brindar un entorno equilibrado y se desarrolle individualmente $\mathrm{y}$ en Sociedad, determina tarea primordial cumplir todas las acciones de promoción, motivación y conservación de la diversidad y el patrimonio protegido según ley y velar por la sostenibilidad de la Amazonía que es una población lejana de nuestra selva.

Así también, lo afirmó el Programa de Desarrollo de las Naciones Unidas y el Medio ambiente (2016), que significó adoptar medidas para combatir la pobreza, proteger el planeta para garantizar la paz y prosperidad de las personas; son 17 objetivos basados en los Objetivos del Milenio, incluye el cambio climático, desigualdad económica, etc. Significa que el logro de uno implica el éxito del otro.

Según la Autoridad regional ambiental (2017) el PRAA de Ucayali (2017-2021), estableció el horizonte de la política regional según competencias, funciones y acciones buscando el desarrollo sostenible, cuyo objetivo general es "Fortalecer capacidades para el monitoreo de acciones de gestión, adaptación y mitigación del cambio climático " planteó acciones para cumplir objetivos y mejorar el desarrollo regional ucayalino buscando la mejora continua de la calidad de vida de los ucayalinos (ver Fig.1).

\section{Figura 1}

Marco conceptual ambiental

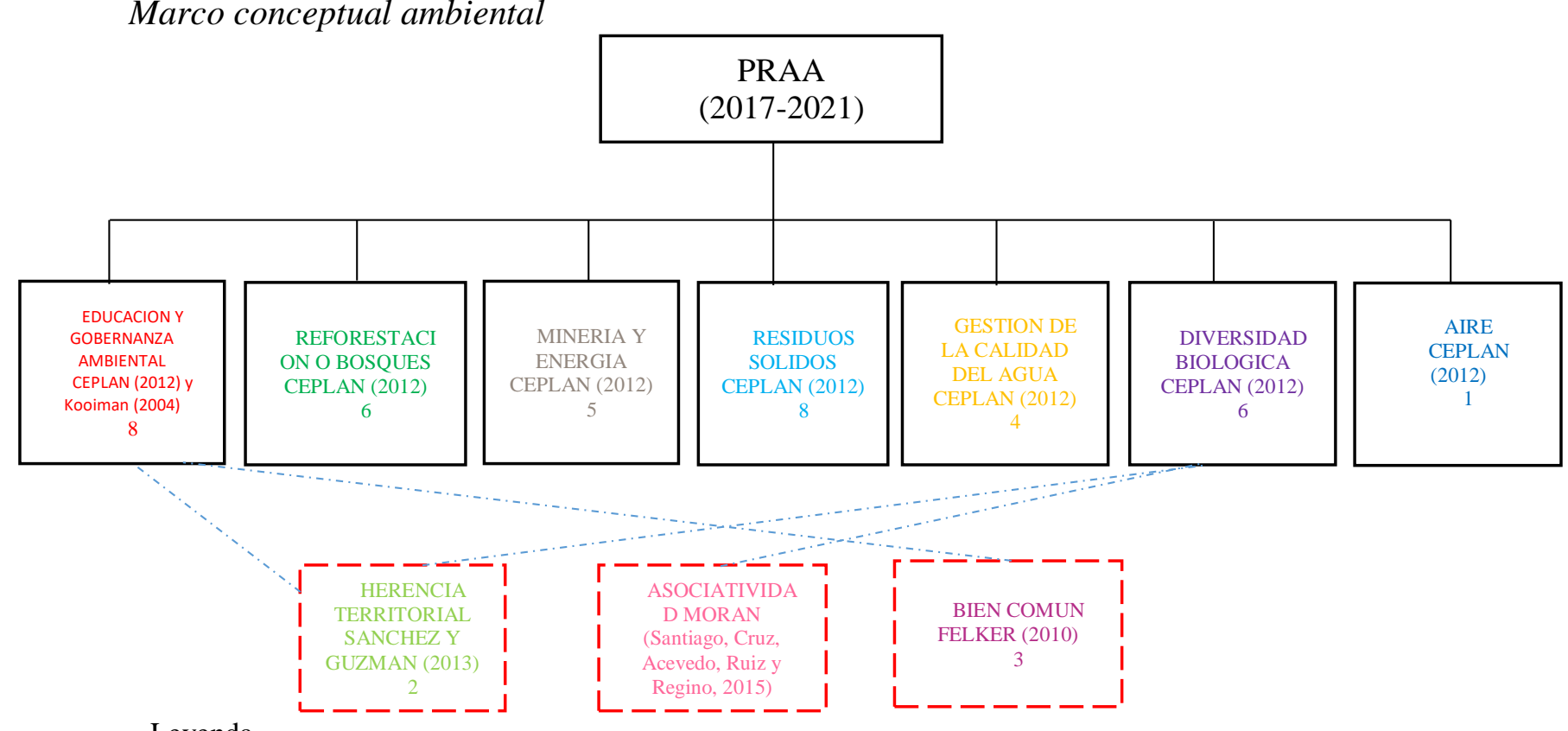

Leyenda 
Ederlinda Ortega Casique.

ISSN 2477-9024. Innova Research Journal (Mayo-Agosto, 2021). Vol 6, No. 2, pp. 255-273

Categoría Apriorística $\quad 1^{---1}$ Categoría Emergente

De acuerdo a Gibson (1979) indicó "la percepción del ambiente es directo, holístico e integral en un marco ecológico donde las propiedades ambientales son percibidas no como puntos diferentes y aislados sino como entidades significativas dentro de un determinado contexto"

Línea estratégica 1 Educación y ciudadanía Ambiental; la gobernanza es "un replantear de las relaciones entre el Estado o gobierno y la sociedad" Kooiman (2004).

Línea estratégica 2 Reforestación; de acuerdo al Centro Nacional de planeamiento Estratégico (CEPLAN, 2012) la migración descontrolada hacia la Amazonía, para acceder a los sistemas productivos agrícolas y pecuarios destruyó alrededor de diez millones de hectáreas de bosques hacia el 2010, sumado al problema de tala y extracción ilegal de madera generó un gran daño ambiental (p.233)

Línea estratégica 3 Gestión de la calidad del agua, según CEPLAN (2012) indicó que se refiere a "vertimiento de aguas residuales no tratadas resultado del uso doméstico o actividades productivas, disposición inadecuada de residuos sólidos en los cursos del agua, existencia de pasivos ambientales mineros y degradación del ecosistema por pérdida de cobertura vegetal u otros" (p.242)

Línea estratégica 4 Minería y Energía; Uno de los pasivos ambientales en el país es ocasionado por la minería en el proceso de excavado abierto y socavones abandonados, relaveras sujetas a erosión, lo dijo CEPLAN (2012), el Sector Energía y Minas es el único que cuenta con normas e información fidedigna sobre pasivos ambientales de la actividad minera y definió como " aquellas instalaciones efluentes, emisiones, restos o depósitos de residuos en la actualidad abandonadas o inactivas y que son un riesgo permanente y potencial para la salud de la población, el ecosistema circundante y la propiedad" (p.245).

Línea estratégica 5 Manejo de residuos sólidos; Según CEPLAN (2012) resumió "la contaminación por residuos sólidos se debe al desorden migratorio e inadecuados hábitos de consumo y educación ambiental precaria; flujos comerciales insostenibles y el inadecuado manejo de los residuos por la mayoría de municipalidades" (p.244)

Línea estratégica 6 Diversidad Biológica; CEPLAN (2012) como una de las estrategias para conservar la diversidad biológica in situ, trató de establecer las áreas Naturales Protegidas(ANP), con el reglamento de la Ley 26839 sobre la Conservación y Aprovechamiento Sostenible de la Diversidad Biológica, precisó las zonas de agro biodiversidad “ orientadas a la conservación y uso sostenible de especies nativas cultivadas por parte de pueblos indígenas, no pueden destinarse para fines distintos a los de conservación de dichas especies y el mantenimiento de las culturas indígenas" es decir conservar la agro diversidad, biocomercio y ecoturismo juntos dentro del contexto (p.236) 
Línea estratégica 7 Aire; Del mismo modo CEPLAN (2012) indicó “el mayor problema ambiental peruano originado por emisiones vehiculares, contaminación por emisiones industriales; incremento del parque automotor con vehículos usados y caótico sistema del transporte son las principales causas de contaminar el aire en las ciudades" (p.240)

Según Sabatier y Jenkins (como se citó en Rodríguez y Espinoza, 2002) brindó una visión basada en el contexto de la región y en ese contexto se busca el fortalecimiento de la capacidad de la sociedad orientado en la protección ambiental (p.7) como en este contexto se busca conservar su naturalidad en cultura y tradición.

De acuerdo a Wilson (2002) manifestó que la educación es básica para la protección ambiental y señaló que "la gran mayoría de nuestros líderes políticos están exclusivamente entrenados en las ciencias sociales y humanidades y tienen muy poco conocimiento de las ciencias naturales" (p.14) eso es lo que sucede actualmente, ya que mucho de los gobernantes obtienen cargos sin la preparación respectiva, mucho menos en la parte medioambiental.

La investigación desarrollada por Sánchez y Guzmán (2013) tomó el nombre de parcialidades, a los grupos sociales unidos por el vínculo de parentesco, en la investigación se refirió a varios grupos étnicos como el Kich'e, caracterizados comúnmente por ser poseedores de extensiones de terrenos y tener una cobertura boscosa de alto nivel de conservación. La Amazonía peruana es rica en sus comunidades nativas como los shipibos conibos, kokamas, Chaminawas los amawacas, la comunidad nativa de Callería de Santa Rita, habitada por shipibos conibos que tienen y mantienen viva la naturaleza primaria que conservan, su cobertura boscosa es heredada de generación en generación, pero sin embargo a veces son atropellados por empresarios privados, que se apoderan de parte de sus tierras ejerciendo su poder económico.

Asociatividad de acuerdo a Morán (como se citó en (Santiago, Cruz, Acevedo, Ruiz y Regino (2015) definió como una gran estrategia en el desarrollo del capital social de grupo y mediante el cual se determinan las actividades de crecimiento, fortalecimiento de las redes participantes del grupo. Asimismo, los recursos asociativos del grupo lo constituyen las relaciones de confianza, reciprocidad y cooperación (p.1173).

El bien común es una filosofía, si bien es cierto se viene trabajando desde hace 14 años y es una propuesta económica basado en la persona en el centro, y tiene a Felker (2010) como representante de esta nueva concepción del bien común, ideológicamente estructurado como un modelo económico centrado en valores como la confianza, honestidad, responsabilidad, cooperación, solidaridad, generosidad o también considerado como sostenibilidad ecológica versus capitalismo generador de la desigualdad y de riqueza.

\section{Metodología}

Corresponde a una metodología hermenéutica y según Vargas (2007), como el mensaje de una realidad profunda develada por Hermes interpretando algo textual, histórico, periodístico, teórico, discursivo, transcripción de entrevistas., etc. Es decir, la realidad concreta vista ya como 
Ederlinda Ortega Casique.

ISSN 2477-9024. Innova Research Journal (Mayo-Agosto, 2021). Vol 6, No. 2, pp. 255-273

texto (p.31). Investigación de enfoque cualitativo, Para Iñiguez (1999) significó involucramiento natural e interpretativo de la realidad a estudiar y trabajar el proceso significó cambio de actitud en tres aspectos: a) cambio en sensibilidad investigadora b) investigación guiada por la teoría porque brinda la conceptualización de los procesos y c) Participación de implicados. Las técnicas utilizadas son la entrevista y el Focus Group y las herramientas utilizadas para llevar a la práctica el presente estudio son la guía de entrevista semiestructurada, no es requisito ser validados se realizó como una forma referencial por los expertos de la Universidad; Los participantes fueron 6 jefes ambientales, 5 líderes de la comunidad y el Focus Group se aplicó a 5 trabajadores del municipio del distrito de Callería.

\section{Tipo y diseño de investigación}

De acuerdo a Ñaupas, Mejía, Novoa y Villagómez (2014) consideró el presente estudio como básico (p.91). El tema ambiental nace por curiosidad de conocer qué pasa dentro del país y lo trascendental es determinar la percepción de la comunidad con respecto al Plan regional de acción ambiental y cómo están articuladas según la normativa, en su ambiente natural y la ciencia permitirá lo que se pretende.

El diseño corresponde al estudio de caso según (Ñaupas et al., 2014) refirió como una forma de búsqueda empírica para estudiar problemas prácticos o específicos, nació en los estudios de tipo médico y sicológico, posteriormente se usó en la sociología y educación, hoy pretende conocer cómo podemos contribuir a solucionar el problema ambiental, Sandoval (1996) refirió el estudio de caso como un proceso caracterizado por ser exhaustiva con variadas perspectivas de lo complejo a lo individual de una determinada Política, Plan o proyecto en un determinado contexto, entender el tema para generar conocimiento.

\section{Escenario de estudio}

Esta investigación se desarrolló en uno de los municipios distritales de la provincia de Coronel Portillo del Gobierno Regional de Ucayali, con entrevistas efectivas en ambientes de las jefaturas ambientales de cada distrito en particular en los distritos de Callería, Yarinacocha, Manantay, Campoverde, Nuevo Requena y el distrito de Masisea, la entrevista al jefe ambiental de Masisea tuvo lugar en la ciudad de Pucallpa producto de una coordinación con su persona. La mayoría de líderes de las comunidades fueron entrevistados en sus casas por la lluvia imperante del día y así se cumplió la realización de la entrevista y dos entrevistados en sus chacras; el Focus Group tuvo lugar en el campus de Maestranza de los trabajadores de la Municipalidad del distrito de Callería con sede principal ubicado en el centro urbano de la ciudad de Pucallpa, distrito de Callería.

\section{Participantes}

Los participantes fueron seis jefes ambientales de los seis municipios distritales de la Región correspondiente a los distritos de Callería, Yarinacocha, Manantay, Campoverde, Nueva Requena y Masisea. Asimismo, cinco líderes de las comunidades y el Focus Group con cinco trabajadores del distrito de Callería, con las siguientes características: a) ser especialistas 
ambientales del distrito con experiencia en temas ambientales, b) ser pobladores de la Región conocedores del tema ambiental, c) Ser dirigentes activos propios del lugar.

\section{Técnicas e instrumentos de recolección de datos}

Técnica, de acuerdo Iñiguez (1999) señaló como técnicas a los procedimientos específicos para el recojo de la información. El instrumento es un documento flexible, estandarizado y moldeable según el avance del trabajo de recolección de datos Hernández (2018). Las técnicas son la entrevista a profundidad y el Focus Group, según Cifuentes (1998) señaló que no es una fuente, sino combina fuentes diversas. Asimismo, señala que la entrevista aborda comportamientos verbales en la extracción de la información sobre las experiencias vividas.

Sandoval (2002) refirió se utiliza haya o no elementos contenidos en los parámetros y criterios de evaluación claves en el cumplimiento de objetivos de un proyecto o programa para el funcionamiento de la institución a estudiar.

Los instrumentos utilizados son: la guía de entrevistas semiestructuradas que plantea algunas preguntas y con posibilidad de plantear otras según transcurra la entrevista para los Jefes Ambientales de cada distrito de la provincia; otra guía de entrevista a los líderes de las comunidades y el guion para el Focus Group dirigido a los trabajadores del distrito de Callería.

\section{Método de análisis de información}

La información obtenida del trabajo de campo, fue grabada, cumpliendo el proceso, algunos entrevistados fueron bastante elocuentes al responder, la mayoría solo respondía con frases cortas, asimismo al escuchar el audio en el caso de las entrevistas realizadas el sábado 19 de octubre, no eran tan claras por la lluvia imperante que duró todo el día y en el caserío la mayoría tiene el techo de sus casas de calaminas y cuando llueve hace mucho ruido cosa que impidió el escucharlos de manera clara, así ocurrió con las cuatro entrevistas llevadas a cabo en este mismo día y en el momento de transcribir fue lo más tedioso lo que tomó tiempo, se codificó, se redujeron los datos que fueron re categorizados y ordenados, el tiempo tomado fue de casi un mes.

\section{Resultados}

De acuerdo al objetivo general, se pudo determinar que se viene trabajando con diversos planes el Institucional, Plan Regional Ambiental de Acción Ambiental y los Planes operativos que aparentemente cumplen con objetivos y metas según lo planificado, pero el avance es lento y existe tan solo una acción que incluye a las comunidades nativas. Aunque algunos o la mayoría no tenía a la mano el PRAA. Y según lo manifestado por dos de los jefes ambientales entrevistados.

"Aquí se está propiciando al cambio de mentalidad de los agricultores para que no ejerzan la quema de árboles para hacer sus chacras porque ello influye en el cambio climático al igual que no a la tala de árboles." 
Con respecto a la línea estratégica 1: Educación y gobernanza, se vienen tomando acciones estratégicas, con programas de concientización y sensibilización, pero solo es por campañas y en ciertos distritos. No es constante, resultando inadecuado si se pretende seguir avanzando, la difusión no se hace por todos los medios, las respuestas difieren dependiendo del lugar, en la zona colindante al departamento de Huánuco que corresponde al distrito de Nueva Requena, donde se ha venido trabajando de manera ordenada y planificada. Mientras que en Callería se vienen desarrollando estas actividades en colegios y coordinando con las universidades para los trabajos en conjunto, pero no hay ordenanzas ni presupuesto para las acciones de manera permanente. Los entrevistados señalan la importancia de la educación ambiental.

"Acciones ambientales desarrolladas con la población (No quemar, No talar árboles, No arrojar los residuos al rio)"

"Toda acción debe enfatizar la educación"

De acuerdo a la línea estratégica 2: Reforestación, se trabaja en el distrito de Nueva Requena y comunidades nativas recuperando bosques degradados por la palma aceitera con la preparación de la tierra y sembrío del cacao, pero esto ocurre en la zona alta de Ucayali, más no en la baja y tampoco es uniforme y según indican recién están en proceso de planificación local e iniciando una nueva gestión. En el distrito de Callería tienen ordenanza sobre las áreas verdes y las declaradas intangibles como las avenidas, plazas, parques y caños naturales, están enfocados en la mitigación a través de las áreas verdes urbanas y el tema paisajístico, no tienen bosques ni productores de bosque porque todo es área urbana pero sí están en planes de hacer sus propios bosques recuperando algunos espacios.

"Recuperación de áreas degradadas (pastizales, terrenos con pendientes de reforestación con árboles maderables" (bolaina en 4 años)

"Lo que hacemos es recuperar los bosques degradados por la siembra de Palma aceitera. En eso estamos apoyando"

Con respecto a la Línea estratégica 3: Minería y Energía, según su jurisdicción los municipios desarrollan normativas autorizando la concesión de extracción de material de construcción, existe poca extracción de metales como el oro, que solo hay en determinados lugares como Nueva Requena y Masisea, pero el Ministerio de Energía y Minas (MINEM) ente rector y La Autoridad Nacional del Agua (ANA), articuladamente acciones con el municipio según las normativas existentes, capacitan en la formalización, el distrito de Callería no cuenta con lugares de extracción de ningún tipo de material.

"Nosotros solo fiscalizamos con respecto al material de acarreo, ellos solicitan la autorización y se trabaja en forma conjunta con ANA, en forma coordinada se sale al campo y verifica y si está disponible y cumple con las características de acuerdo a la evaluación se autoriza." 
En cuanto a la Línea estratégica 4: Manejo de los Residuos Sólidos en el distrito de Callería; Actualmente, dos de las cinco municipalidades cuentan con el Plan Integral de Gestión de los Residuos Sólidos (PIGARS), los demás solo tienen plan de segregación de los residuos sólidos y no cuentan con muchas empresas recicladoras, en el caso de Callería recién tiene una empresa formalizada y otra que está en proceso de formalización y el municipio no establece puentes en el fortalecimiento de las actividades de manera continua y disponer de presupuestos en el desarrollo de las actividades y estén articulados con el factor educación, cuentan con maquinaria adquirido por la municipalidad para realizar el trabajo, pero no se abastece para toda la ciudad, ésta gestión está abocada en solucionar el problema del Botadero Municipal a otra municipalidad como la de Manantay cuenta con cuatro empresas de recojo.

"Enseñanza del manejo de los RRSS orgánicos e inorgánicos en los hogares"

"Las empresas recicladoras quieren que se les dé todo; lo que significa más gastos que beneficios para el municipio"

Resultados con respecto a la Línea Estratégica 5: Gestión de la Calidad del agua en el distrito de Callería, se viene difundiendo por la radio y tv; cuentan con un programa de defensa sanitaria y el programa del uso del agua; además hay un proyecto de saneamiento de agua y desagüe para la comunidad nativa de Callería que el municipio viene desarrollando en el distrito.

“Capacitación del personal, estamos implementando la ley de salud en el trabajo el mismo que abarca capacitación, inducción al personal que se dedica a esta actividad, el equipo necesario, certificados médicos y toda la temática del agua"

Con respecto a la línea estratégica 6: Diversidad Biológica, los entrevistados del distrito y la comunidad manifestaron que a nivel provincial se van a crear las áreas de conservación municipal(ACRM) y se vienen coordinando con los de Servicio Forestal(SERFOR) y solicitando alianzas pero el problema es el presupuesto, se viene trabajando pero no es suficiente, lo hacen de manera muy lenta o al parecer no conocen los procedimientos de actuación ante éste tema, es sabido que hay varios lugares para conservar pero hasta hoy no se ha hecho efectivo al igual que el biocomercio y el ecoturismo están aletargados pudiendo ser explotados. Según lo manifestado.

"Dentro de su jurisdicción tiene algunas lagunas el año pasado hemos trabajado dentro de Pucallpillo y se ha estado trabajando buscando cómo realizar el proyecto de conservación de esta laguna o área de protección porque la municipalidad no tiene competencia para asignar esa parte que debemos conservar y se llama la laguna Chanajao destinado al Ecoturismo.”

En la línea estratégica 7: Aire, a nivel del distrito se sabe que hay normas que cumplir y se vienen realizando las primeras acciones dedicadas a este fin. En Callería se están elaborando las normativas para reducir la contaminación del aire producto del transporte y disminución de ruidos; no se controla los ECA, está en proyecto. La mayoría de los entrevistados coinciden en que los habitantes son beneficiados por la poca contaminación del aire por la tenencia de cobertura boscosa. 
Ederlinda Ortega Casique.

ISSN 2477-9024. Innova Research Journal (Mayo-Agosto, 2021). Vol 6, No. 2, pp. 255-273

“Aquí no hay mucha contaminación con respecto a las ciudades que sí lo tienen. Somos una gestión nueva que siempre está buscando formalizar muchas actividades y la administración y poder desarrollar todo el tema ambiental. Tenemos el PLANEFA 2019, Plan EDUCA 2019 tenemos desarrollado el Plan de los Residuos sólidos que son los instrumentos para poder desarrollar y al que nos hemos adecuado y siempre estamos informando al ministerio lo que se está haciendo."

De acuerdo a los resultados obtenidos basados en las líneas estratégicas que son las categorías apriorísticas y como resultado de las entrevistas y el Focus Group, se obtuvo en principio nueve categorías emergentes y que son: Liderazgo comunitario; Herencia territorial etnias, Asociatividad comunitaria, participación, presupuesto, Bien común y vías de comunicación o transporte prioritario, motivación. Se eliminaron las categorías apriorísticas por ser transversales a las líneas estratégicas del plan.

Como resultado final del análisis se tiene las siguientes categorías emergentes:

Herencia territorial étnicas "Madre tierra la que nos da el alimento"

Asociatividad comunitaria "Todos debemos de pertenecer a una asociación"

Bien común "Basta un poco más de tiempo para nosotros tener un medio ambiente más

limpio y donde las relaciones sociales estén más fortalecidas para tener una vida mucho mejor"

\section{Discusión}

Si bien es cierto, que los antecedentes considerados muestran la preocupación a nivel mundial en el tema ambiental y sus consecuencias orientando acciones presentes pensando en el futuro de la humanidad y según los resultados se puede decir que se ha venido trabajando parcialmente según lo planificado. Escobar (como se citó en Rodríguez y Espinoza, 2002) considerando al Estado como promotor y motivador de todas sus fuerzas sociales unidas en las acciones de preservación, conservación, restauración y uso sostenible del medio ambiente, paralelamente debe evaluarse los avances asimismo Gibson (1979) la percepción es directa y única en el contexto, diferente a otro contexto, es decir, no es lo mismo en la ciudad como en el campo, así lo consideran estudios de Latinoamérica y el Caribe, una política o un plan involucra al país y es articulada a los planes en la ciudad y en el campo, sostiene que todo accionar no es aislado, ni responder a intereses ajenos, buscando preservar y conservar las riquezas naturales propias de la región, mientras la postura de Sabatier y Jenkins, OCDE (como se citó en Rodríguez y Espinoza, 2002), buscó fortalecer la capacidad de la sociedad para la protección ambiental en el contexto, respetando su naturalidad, cultura y tradición. En Ucayali el PRAA, resulta del trabajo en conjunto de sus instituciones y la sociedad de la región Ucayali, muestra que se pueden obtener mejores resultados si se proponen, según lo manifestado por la Red Internacional Published on Servindi (2015) comprobaron que en Perú, se desarrollan diversas actividades como conferencias, fórum y debates para obtener información de interés común del país y proponer mejoras e iniciativas pero éstas no llegan a la región Ucayali, por lo tanto, las autoridades no se implican en el tema, no toman decisiones acertadas, desconocen el tema, ni hay involucramiento en las acciones o son indiferentes sin saber que repercutirá en beneficio o perjuicio de su población y comunidades 
nativas dentro del territorio, además se desconoce la participación de las empresas públicas y privadas, si tienen normas o cuentan con estrategias participativas.

Según la línea estratégica 1. Educación y gobernanza, se viene trabajando con programas de concientización y sensibilización en las entidades educativas del distrito, que es insuficiente por su efectividad solo en campañas y en algunos distritos, es temporal, con difusión parcial, sin normativas, será imposible lograr el involucramiento de la gente y poco se podrá alcanzar si no seguimos a Koimam (2004) quien manifestó para que la gobernanza sea óptima hay que replantear las relaciones entre el Estado, gobierno y la sociedad en general mientras, que en otros países según los trabajos especializados de Tabares y Vera (2017) quienes consideran normas y fomento del medio ambiente y desarrollo sostenible en los países respetando valores y culturas propias así la gobernanza estará presente en la parte operativa buscando involucramiento y educación como herramienta clave.

En la línea estratégica 2. Reforestación o bosques en el distrito y comunidades nativas se recuperan los bosques degradados por la coca y palma aceitera remplazándola por el cacao y árboles en la zona alta pero no en la baja de Ucayali, existe cambio de gestión e inicio del proceso de planificación local. Cuentan con ordenanzas respecto a las áreas verdes y áreas declaradas intangibles. El municipio viene elaborando proyectos de recuperación de bosques coincidiendo con el teórico Fiorino (como se citó en Rodríguez y Espinoza, 2002) consideró que el plan puede ser un instrumento articulado u otros complementos para que la ejecución sea efectiva poniendo como ejemplo si se prohíbe la tala de un árbol, será más efectivo si es difundido por todos los medios para llegar a los hogares y garantizar su cumplimiento y si todos tienen conocimiento de ello, como parece haber ocurrido aquí en el contexto o no todos contaban con la norma o ésta no ha sido debidamente difundida, ni cumplida, tampoco fue promovida.

Del mismo modo, para la línea estratégica 3. Minería y Energía, bajo esta línea, los municipios trabajan en sus normativas de autorización para concesión de extracción de material de construcción y no priorizan la extracción de oro y otros metales favoreciendo la informalidad aunque en menor proporción, otro factor negativo es la extracción del oro a lo largo del río Ucayali, lugares difíciles de ejercer el control, sin un presupuesto adecuado, en el municipio de Callería, se viene trabajando las normativas y capacitando en formalización de las empresas pero aquí no existen lugares de extracción de material de construcción tampoco de oro. Según Santos (2017) expuso los beneficios que brindan los ecosistemas mejorando la calidad de vida, propone el uso correcto y su repercusión en la cultura de las comunidades locales, indicando mayor efectividad si va acompañado de responsabilidad como resultado de la planificación.

De acuerdo a la Línea Estratégica 4: Manejo de los Residuos Sólidos, en el distrito de Callería, solo dos, de las cinco municipalidades cuentan con el Plan Integral de Gestión de los Residuos sólidos (PIGARS), los demás solo cuentan con plan de segregación de los residuos sólidos y pocas empresas recicladoras. Callería tiene una empresa formalizada y otra por formalizar, falta establecer puentes para fortalecerla, hacerla continua y permanente, con presupuesto y articulada al factor educación como respuesta a una planificación efectiva, cuenta con maquinaria insuficiente para atender la ciudad; la gestión está concentrada en erradicar el botadero municipal, problema originado por el crecimiento poblacional al centro de la ciudad, así 
Ederlinda Ortega Casique.

ISSN 2477-9024. Innova Research Journal (Mayo-Agosto, 2021). Vol 6, No. 2, pp. 255-273

lo manifiesta CEPLAN (2012) considerándolo como un factor de contaminación, así como inadecuados hábitos de consumo, educación ambiental precaria, mal manejo de los residuos por la mayoría de municipalidades, etc., resultando prioritario incidir en la educación además, los estudios de Boto y Mellado (2018) se avanzó en las normativas en el Principado de Asturias en residuos y contaminación, priorizaron temas de protección y control ambiental industrial, actuación de funcionarios medioambientalistas, uso de insecticidas y del agua, etc. Es decir, se avanzó en normas para contrarrestar las consecuencias ambientales, sin embargo, son los primeros ocasionadores de la contaminación ambiental por poseer grandes industrias, en Callería la atmósfera no está tan contaminada porque no cuentan con industrias, pero sí el uso de insecticidas en los sembríos de la palma aceitera que degradan los suelos y venenos en el desarrollo de la agricultura y uso de suelos y la pesca.

En la Línea Estratégica 5: Gestión de la Calidad del agua, Callería cuenta con el programa de defensa sanitaria y uso del agua, los que son difundidos por los medios más vistos que son la radio y tv; En Callería no se puede consumir el agua sin tratar, aquí el municipio está implementando un proyecto de saneamiento de agua y desagüe para la comunidad nativa de Callería y según las investigaciones de Cortés (2019), resaltó la importancia del medio ambiente, el agua y sus efectos, suelos degradados que deterioran el paisaje, brinda las estrategias de prevención para promover el desarrollo sostenible lo que convendría emular para todas las comunidades.

En la Línea Estratégica 6: Diversidad Biológica, en Callería se viene intentando incrementar áreas para la conservación municipal (ACRM), como la de la Isla del Chanajao que se encuentra en el centro del rio Ucayali, donde habitan muchas aves silvestres y está rodeada de delfines rosados, en coordinación con SERFOR se pretende promover el desarrollo de actividades de biocomercio y ecoturismo sostenible, aquí el problema es el presupuesto, se viene trabajando pero es insuficiente, lento y no saben los procedimientos de actuación, existen otros lugares para conservar pero no se efectiviza; El biocomercio y ecoturismo están aletargados pudiendo ser explotados quizá por la falta de compromiso de las autoridades o por falta de capacidad en la gestión que resulta contraproducente a lo referido por Tabares y Vera (2017) "cuidar el medio ambiente significa compromiso, responsabilidad, cumplir acuerdos, desarrollar políticas, planes y programas y ejecutarlos" y no se estaría buscando la sostenibilidad porque estarán incumpliendo las acciones a nivel nacional, como lo indicó CEPLAN (2012) establecimiento e incremento de las ANP, al darse las nuevas ACRM estaríamos cumpliendo con la protección, conservación y promoviendo el desarrollo de los habitantes de la isla del Chanajao y sus especies nativas que allí habitan, pero en Callería no habitan ninguna comunidad nativa.

En la Línea Estratégica 7: Aire, según Amador y Mendoza (2018) coincidieron al manifestar compromiso de responsabilidad y cuidado del MA, cumplir los compromisos adoptados en RIO+20 y COP21, en el distrito de Callería hay normas para reducir la contaminación del aire producto del transporte y disminución de ruidos; no se controla los ECA, porque la población no se encuentra preocupado en este tema por contar con cobertura boscosa que descontamina el aire y no lo consideran un problema cosa que considero que sí se debe iniciar el cuidado con acciones preventivas evitando mayores riesgos.

Esta obra se comparte bajo la licencia Creative Common Atribución-No Comercial 4.0 International (CC BY-NC 4.0) Revista de la Universidad Internacional del Ecuador. URL: https://www.uide.edu.ec/ 


\section{Conclusiones}

El distrito de Callería está en el centro de la ciudad de Pucallpa que es la capital de la región muy comercial, que, si bien cuentan con el PRAA, algunas normativas articuladas a nivel regional y nacional pero su ejecución y puesta en marcha no es cumplida o se cumple en forma parcial es decir en algunas zonas o encuentra limitantes como falta de presupuesto para las acciones cosa que no puede suceder si consideramos que Perú es un país descentralizado.

De acuerdo al objetivo general del PRAA, Que busca el fortalecimiento de capacidades y monitoreo de las acciones de gestión, adaptación y mitigación del cambio climático, pero éstas no son controladas ni monitoreadas para comprobar los avances y de acuerdo a los resultados las autoridades puedan tomar decisiones.

El análisis se realizó basado a las líneas estratégicas del PRAA, que sirvió para el proceso de categorización que serían las categorías apriorísticas del estudio.

Considerando las categorías emergentes (herencia territorial, asociatividad comunitaria y bien común), derivadas del presente estudio se está considerando una propuesta de acciones para fortalecer las líneas estratégicas existentes, promoviendo la inclusión social y la mejora de la calidad de vida de las comunidades nativas del distrito de Callería en alianza con el empresariado, sin ejercer presión sobre los sistemas ecológicos y naturales y así contribuir con el futuro de las generaciones de las comunidades nativas y lograr que se desarrollen con dignidad y solidaridad en la región que habitan. No considero oportuno agregar una nueva línea estratégica debido a la burocracia, pero sí se podría trabajar complementando las acciones de manera más rápida si se trabaja a nivel regional, las acciones consideradas están en la tabla 1.

\section{Tabla 1}

Acciones a implementa y recursos

\begin{tabular}{|c|c|c|}
\hline Acciones & Recursos & Responsable \\
\hline Herencia territorial & stand para feria & ARAU \\
\hline $\begin{array}{l}\text { 1. Realizar eventos de interculturalidad de tal manera } \\
\text { que promuevan sus artesanías hechas por la } \\
\text { comunidad al igual que otros productos }\end{array}$ & $\begin{array}{l}1 \text { gestor ( } 2 \text { veces al } \\
\text { año) }\end{array}$ & \\
\hline $\begin{array}{l}\text { 2. Campañas informativas de información ambiental } \\
\text { para las comunidades de tal forma que contribuyan en } \\
\text { la vida regional o municipal }\end{array}$ & 2 capacitadores & ARAU \\
\hline Asociatividad & medios, difusión & ARAU \\
\hline $\begin{array}{l}\text { 3. Implementar mecanismos de comunicación que } \\
\text { brinde información Sobre los servicios de la } \\
\text { asociación }\end{array}$ & boletines al mes & \\
\hline $\begin{array}{l}\text { 4. Promover la asociatividad e integración del } \\
\text { empresariado para los Proyectos productivos bajo este } \\
\text { medio }\end{array}$ & $\begin{array}{l}1 \text { gestor y equipo } \\
\text { de trabajo }\end{array}$ & ARAU \\
\hline
\end{tabular}


Ederlinda Ortega Casique.

ISSN 2477-9024. Innova Research Journal (Mayo-Agosto, 2021). Vol 6, No. 2, pp. 255-273

\begin{tabular}{lcl}
\hline Acciones & \multicolumn{1}{c}{ Recursos } & Responsable \\
\hline Bien común & 1 equipo de trabajo & ARAU \\
$\begin{array}{l}\text { 5. Realizar un diagnóstico con la finalidad de conocer } \\
\text { las necesidades reales de la comunidad }\end{array}$ & & \\
$\begin{array}{l}\text { 6. Poner en marcha programas de ocio y tiempo libre } \\
\text { para los jóvenes y Actividades deportivas }\end{array}$ & 1 equipo de trabajo & ARAU \\
$\begin{array}{l}\text { 7. Diseñar programas de educación ambiental para las } \\
\text { instituciones en las comunidades }\end{array}$ & 1 equipo de trabajo & ARAU \\
\hline
\end{tabular}

Elaboración propia

Según el PRAA, en la línea estratégica, Educación y Gobernanza se considera como acción 1.2.2 la implementación del manejo forestal comunitario teniendo como indicador cantidad de comunidades que implementan el Manejo forestal Comunitario. Que es la única acción definida con respecto a estos grupos originarios de la región y que merecen mayor inclusión y empoderamiento dentro del territorio originario, es por ello, el pretender el fortalecimiento de las líneas para el empoderamiento de las comunidades nativas en búsqueda del bienestar social mediante la educación y diversidad biológica aquí considero algunas ventajas de complementar la propuesta de acciones para el PRAA según la tabla 2.

\section{Tabla 2}

Ventajas de las acciones propuestas

\begin{tabular}{|c|c|}
\hline Ventajas & Características \\
\hline Integración Social & $\begin{array}{l}\text { Acciones dinámicas, inclusivo e integrador resultado de una buena } \\
\text { planificación }\end{array}$ \\
\hline Empoderamiento & Las comunidades nativas son los actores principales \\
\hline Participación & $\begin{array}{l}\text { Fomento y fortalecimiento de la participación de cada miembro de la } \\
\text { comunidad como ciudadanos }\end{array}$ \\
\hline Sostenibilidad & $\begin{array}{l}\text { Búsqueda de la sostenibilidad mediante la educación para avanzar en el } \\
\text { desarrollo. }\end{array}$ \\
\hline Comunicación & $\begin{array}{l}\text { Mayor comunicación e información para alcanzar el consenso e } \\
\text { implicación en las mismas ya que actualmente es un derecho de la } \\
\text { ciudadanía a la consulta, iniciativas y transparencia en la gestión } \\
\text { condición necesaria para la eficacia y lograr lo sostenible. }\end{array}$ \\
\hline Involucramiento & $\begin{array}{l}\text { Presencia de las autoridades y personas para implementar, hacer } \\
\text { seguimiento y monitoreo de las acciones }\end{array}$ \\
\hline Valor & Respeto a la cultura y tradición \\
\hline
\end{tabular}




\section{Referencias Bibliográficas}

Amador, M. y Mendoza, A. (2018). Evaluación del Plan Nacional de Desarrollo Humano en el cumplimiento del derecho sostenible que estipula el Marco Internacional de los compromisos de Rio +20 , periodo 2012-2016. [Tesis de maestría, Universidad Nacional Autónoma de Nicaragua]. http// repositorio.unam.edu.ni/id/eprint/7860

Autoridad Regional Ambiental de Ucayali. (2017). Plan Regional de Acción Ambiental (20172021) Ucayali. Pucallpa: Gobierno Regional de Ucayali.

Boto, A. y Mellado, L. (2018). Derecho y Políticas ambientales en Asturias. Dret Ambiental, IX(2 y 1), 1-10. Recuperado el 17 de 04 de 2019

Caicedo, P. y Morvelli, V. (2016). Evaluación de Impacto ambiental y su relación con la Evaluación Ambiental Estratégica y el Ordenamiento Territorial en proyectos de inversión de gran escala: Caso Conga e Inambari. Lima, Lima, Perú: Pontificia Universidad Católica del Perú.

Centro Nacional de planeamiento Estratégico. (2012). Plan Estratégico de Desarrollo Nacional. Lima: CEPLAN.

Chiarelli, R. (2018). Planificación Estratégica y Desarrollo Territorial. Lima, Perú.

Cifuentes, M. (1998). Diseño de Proyectos: Modalidades, estrategias y técnicas. Argentina: Lumen Humanitas.

Cortés, C. (2019). Evaluación Ambiental Estratégica para la formulación de políticas de ordenamiento territorial de Ecosistemas. Estudio de Caso: Complejo de Páramos Jurisdiccionales- San Turbán-Berlín. Colombia.

Echevarría, F. Guillinta, W. Sifuentes, D. y Zapata, R. (2018). Planeamiento Estratégico de la Región Loreto. [Tesis de grado, Universidad Católica del Perú]. http://tesis.pucp.edu.pe/repositorio/handle/123456789/12580

Escobar, A. (1999). El final salvaje, naturaleza, cultura y política en la Antropología contemporánea. Santa Fe, Bogotá, Colombia.

Felker, C. (2010). La Economía del Bien Común. Madrid.

García J, D. Inga J, A. y Párraga, E. (2018). Planeamiento Estratégico de la Región Junín. [Tesis de maestría, Pontificia Universidad Católica del Perú]. http://tesis.pucp.edu.pe/repositorio/handle/123456789/11979

Gerencia Regional de Planeamiento, Presupuesto y Acondicionamiento Territorial de la Región Ucayali. (2015). El Plan Regional de Desarrollo Concertado. Pucallpa.

Gibson, J. (1979). The ecological Approach Visual Perception.

Hernández, R. (2018). Metodología de la Investigación: Las Rutas Cuantitativas, Cualitativa y Mixta. (E. I. C.V., Ed.) México, México: Mc Graw Hill Education Ed.

Intergovermental Science Police Plattform on Biodiversity and Ecosytem Services. (06 de 05 de 2019). Actualidad Ambiental. La naturaleza en declive peligroso.

Iñiguez, L. (1999). "Investigación y Evaluación Cualitativa: Bases teóricas y conceptuales". En atención primaria (Vol. 23). Barcelona, España: Universidad Autónoma de Barcelona. Recuperado el 18 de mayo de 2019 
Ederlinda Ortega Casique.

ISSN 2477-9024. Innova Research Journal (Mayo-Agosto, 2021). Vol 6, No. 2, pp. 255-273

Kooiman, J. (2004). Gobernar en Gobernanza (Vol. 16). Ed.

Martínez, S. y Ortiz, C. (2019). Auditoría Ambiental de cumplimiento en la empresa de servicios de rastro y plaza de ganado al Plan de manejo ambiental en el periodo 2017-2018.ñ. (U. d. Azuay, Ed.) Azuay, Colombia: Universidad de Azuay.

Ministerio del Ambiente. (8 de 06 de 2009). minam.gob.pe. Obtenido de minam.gob.pe.

Ministerio del Ambiente. (2013). Constitución Política del Perú. Lima, Lima, Perú.

Monteiro, M. B., do Rosário Partidário, M., \& Meuleman, L. (2018). A comparative analysis on how different governance contexts may influence Strategic Environmental Assessment. Environmental Impact Assessment Review, 72(September), 79-

87. https://doi.org/10.1016/j.eiar.2018.05.010

Morán, J. (2010). Capital social: Las redes sociales y su impacto sobre el desarrollo socio económico. Obtenido de www.eumed.net/librosf/860/

Ñaupas, H. Mejía, E. Novoa, E. y Villagómez, A. (2014). Metodología de la Investigación (4ta ed. ed.). Bogotá, Colombia: Ediciones de la U.

Organismo de Evaluación y Fiscalización Ambiental. (15 de 09 de 2018). Ley N² 29325. Lima, Perú.

Programa de Desarrollo de las Naciones Unidas y el Medio ambiente. (2016). El desarrollo sostenible en la práctica. (ONU, Ed.) ONU.

Published on Servindi. (29 de 09 de 2015). El Perú: Desafío de la Evaluación Ambiental Estratégica (EAE). 3. Servindi comunicación Intercultural.

Rodríguez, M. y Espinoza, G. (2002). Gestión ambiental en América Latina y el Caribe. Washington D.C.: Banco Interamericano de Desarrollo ed.

Sabatier, A. \& Jenkins, S. (1993). Police change and learning: An advocaci Coalition Approach.

Sánchez, A. y Guzmán, D. (2013). Entre la teoría y la práctica de la conservación de los recursos naturales: Las parcialidades de Totonicapán, Guatemala (Vol. 26). Guatemala.

Sandoval, A. (1996). Investigación Cualitativa. Bogotá, Colombia: ARFO.

Sandoval, A. (2002). Investigación Cualitativa (Vol. 4). (E. I.C.ICFES, Ed.) Bogotá, Colombia: ARGO editores e Impresores Ltda.

Santiago, P. Cruz, C. Acevedo, A. Ruiz, A. y Regino, J. (Enero- Junio de 2015). Asociatividad de la Competitividad en la Agroindustria de OAXACA. Revista Mexicana de Agronegocios, 36, 1116-1117. https://www.redalyc.org/articulo.oa?id=14132408003

Santos, M. (2017). Global justice and environmental governance: an analysis of the Paris

Agreement. Revista Brasileira de Política Internacional, 60(1), e008. Epub February 20,

2017.https://doi.org/10.1590/0034-7329201600116

Tabares, V. y Vera, L. (2017). Evaluación del Plan del Distrito de Manejo Integrado (DMI), En el Municipio de La Palma- Cundinamarca y Propuesta de mejoramiento. (U. D. Caldas, Ed.) Bogotá, Colombia: Universidad Distrital Francisco José de Caldas - Facultad de Medio Ambiente y Recursos Humanos.

Vargas, X. (2007). ¿Cómo hacer investigación cualitativa? Una guía práctica para saber qué es la investigación en general y cómo hacerla, con énfasis en las etapas de la investigación cualitativa. Jalisco, México: ETXETA.

Wilson, E. (2002). Gestión Ambiental en América Latina y el Caribe. (B. I. Desarrollo, Ed.) David Wilk. 
Zúñiga, P. (2018). Evaluación Ambiental Estratégico de la Política del Sector Transporte para la mitigación del cambio climático en Colombia. Colombia. Obtenido de http//hdl.handle.net/1349/6794 